\title{
Delirium: a guide for the general physician
}

\author{
Authors: Oliver M Todd ${ }^{\mathrm{A}}$ and Elizabeth $\mathrm{A}$ Teale $^{\mathrm{B}}$
}

Delirium describes a sudden onset change in mental status of fluctuating course. This is a state of altered consciousness characterised chiefly by inattention or lack of arousal, but can also include new impairment of language, perception and behaviour. Certain predisposing factors can make an individual more susceptible to delirium in the face of a stressor. Stressors include direct insults to the brain, insults peripheral to the brain or external changes in the environment of an individual. Delirium is varied in its presentation, and can be categorised by the psychomotor profile as: hyperactive type (overly vigilant, agitated, often wandersome), hypoactive type (sedate or withdrawn) or mixed types.

KEYWORDS: Clinical management, delirium, detection, prevention, treatment

\section{About delirium}

Overall, prevalence of delirium on admission to hospital ranges between $10 \%$ and $31 \%{ }^{1}$ but estimates vary according to the population measured. Prevalence is highest among those who are frail or patients who are critically unwell, for example patients on intensive care units, following surgery, or at the end of life. Over the age of 80 years, more than one third of those in hospital will experience delirium. ${ }^{2}$ Despite its high prevalence, delirium often goes undetected ${ }^{3}$ and undetected delirium is associated with the highest mortality. ${ }^{4}$

Delirium heralds high risk of falls, longer inpatient stay, postdischarge institutionalisation, accelerated and lasting cognitive decline, and higher mortality. ${ }^{5}$ Perhaps most importantly for the affected person and their family, delirium can be a cause of significant distress. ${ }^{6}$

Delirium is both common and dangerous, but current evidence suggests it is also preventable in about one third of cases, ${ }^{7}$ hence the growing emphasis on the adoption of multicomponent delirium prevention interventions. Delirium prevalence is not bound by specialty ${ }^{2}$ and crosses over to both hospital and community settings. ${ }^{8}$ Given its ubiquity and its heterogeneous presentation, delirium diagnosis and management is the responsibility of all clinicians.

Authors: A geriatrics registrar, Bradford Institute for Health Research, Bradford, UK; ${ }^{\mathrm{B}}$ Clinical senior lecturer in geriatric medicine, Bradford Institute for Health Research, Bradford, UK

\section{Clinical management}

\section{Identify baseline function}

Delirium is characterised by new changes to baseline mental state. Speaking to an informed caregiver or family member to establish the patient's background cognitive function, physical function and independent ability to perform activities of daily living is therefore key to diagnosis. National Institute for Health and Care Excellence (NICE) guidelines recommend early identification of patients who are vulnerable as a result of non-modifiable risk factors, which lower the threshold for developing delirium (Box 1). ${ }^{9}$ Dementia is the most significant of these and the more advanced the dementia, the higher the risk. ${ }^{10}$ Dementia can be identified on admission with a high degree of accuracy (C-statistic 0.93$)^{11}$ using the Informant Questionnaire of Cognitive Decline in the Elderly (IQCODE). ${ }^{12}$

\section{Suspect delirium early}

Delirium is a state of altered consciousness, manifested primarily as impaired arousal (altered level of consciousness) and inattention (altered content of consciousness). ${ }^{13}$ It is a clinical diagnosis and requires fulfilment of five criteria according to the Diagnostic and Statistical Manual of Mental Disorders, 5 th edition (Box 2). ${ }^{14}$ The fifth criterion requires that a cause be found and this demands a full medical evaluation. A noteworthy exception may be where investigation is not appropriate (for example in palliative care).

Screening tests attempt to identify key features of delirium without which it is unlikely to be present. These tend to be simple, short and easy to embed into daily clinical practice. Asking a friend or relative the question 'Do you think [the patient] has been more confused lately?' has an $80 \%$ sensitivity for detecting delirium. ${ }^{15}$ Inattention, a cardinal feature of

\section{Box 1. Predisposing factors in delirium}

$>$ Dementia
$>$ Frailty
$>$ Drug/alcohol dependence
$>$ Sensory impairment
$>$ Polypharmacy
$>$ Multimorbidity
$>$ Male sex
$>$ Depression


Box 2. DSM-5 criteria for the diagnosis of delirium ${ }^{1}$

1 Disturbance in attention and awareness

2 Onset over a short period (hours to days) with tendency to fluctuate over the course of the day

3 Change in cognition or perceptual disturbance

4 Changes not accounted for by an established underlying disease or coma

5 Evidence from the history, examination or investigations that the disturbance is caused by a general medical condition.

DSM-5 = Diagnostic and Statistical Manual of Mental Disorders, 5th edition

delirium, can be quickly identified by asking someone to recite the months of the year backwards. Failure to reach July on this test has a sensitivity of $83 \%$ and specificity of $91 \%$ for picking up delirium although specificity is reduced in patients with background dementia. ${ }^{16}$

Delirium is a syndrome, therefore it is not usefully reducible to a single deficit, and a combination of tests demonstrates higher discrimination. The 4 As test (4AT) presents one such battery: it is well validated across different settings, brief and requires no training (Fig 1). ${ }^{17,18}$ Where there is uncertainty about whether cognitive deficits are new, such patients should be treated as if delirium is present until evidence to the contrary is available.

The fluctuating nature of delirium means that serial assessment is required. One difficulty is that repetition of cognitive assessments will be affected by a practice effect. Therefore, alongside cognitive tests, NICE guidelines recommend daily assessment of perception, and physical and social behaviour. ${ }^{9}$ Perception can be measured by checking orientation to time, place and person, as well as screening for auditory and visual hallucinations.

Physical and social behaviour can be categorised as hyper- or hypo-active delirium. Hyperactive behaviour, by its nature, demands attention of nursing staff and, therefore, tends to be diagnosed earlier, with a better prognosis. Hypo-active behaviour is characterised by reduced arousal; hence, it can be challenging to identify. Hypo-active delirium is also more common, particularly among older people, and carries with it a poorer prognosis. ${ }^{19}$ Bedside observations of abnormal hand movements, 'aimlessly picking at bedclothes' or 'plucking at the air', show a high specificity for delirium of both hyper- and hypo-active subtypes. ${ }^{20}$

\section{Identify and treat triggers}

A thorough history is required to elicit possible delirium precipitants, which themselves can be numerous and varied in one individual (Table 1). Attention should be paid to preventing modifiable factors, and attenuating the impact of non-modifiable risk factors. Patients with delirium often struggle to follow the commands of a traditional neurological exam, yet careful observation at the bedside may identify signs attributable to the underlying aetiology.

A full assessment of prescriptions, recent changes, over-thecounter or homeopathic remedies and adherence is required. Many medicines have deliriogenic side effects, particularly those with anti-cholinergic profiles (Box 3). ${ }^{21,22}$ Sudden withdrawal of opiates, benzodiazepines and other sedatives may also cause delirium and if these medications are to be stopped, it should be done gradually.

\section{Table 1. Triggers for delirium}

\section{Precipitating}

factors
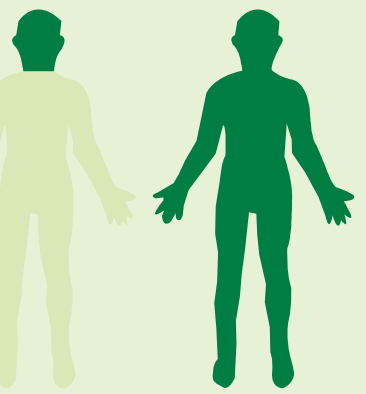

Peripheral

Central

Direct brain trauma

Stroke

\section{haemorrhage}

Subdural

\section{Epileptic}

seizure

Encephalitis

Encephalitis

A

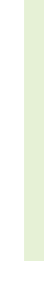

Box 3. Medication classes with known delirium
potential ${ }^{22}$
$>$ Neuroleptic
$>$ Opioid
$>$ Benzodiazepine
$>$ Anti-histamine
$>$ Dihydropyridine
$>$ H2 receptor antagonist
$>$ Cardiac glycoside
$>$ Steroid
$>$ NSAID
$>$ Tricyclic antidepressant
$>$ Anti-parkinson
NSAID = Non-steroidal anti-inflammatory agent




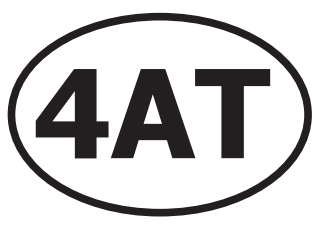

Assessment test for delirium \& cognitive impairment
Patient name:
Date of birth:

Patient number:

Date: $\quad$ Time:

Tester:

CIRCLE

\title{
[1] ALERTNESS
}

This includes patients who may be markedly drowsy (eg. difficult to rouse and/or obviously sleepy during assessment) or agitated/hyperactive. Observe the patient. If asleep, attempt to wake with speech or gentle touch on shoulder. Ask the patient to state their name and address to assist rating.

Normal (fully alert, but not agitated, throughout assessment)
Mild sleepiness for $<10$ seconds after waking, then normal
Clearly abnormal

[2] AMT4

Age, date of birth, place (name of the hospital or building), current year.

\author{
No mistakes \\ 1 mistake \\ 2 or more mistakes/untestable
}

\section{[3] ATTENTION}

Ask the patient: "Please tell me the months of the year in backwards order, starting at December." To assist initial understanding one prompt of "what is the month before December?" is permitted.

Months of the year backwards

\section{[4] ACUTE CHANGE OR FLUCTUATING COURSE}

Evidence of significant change or fluctuation in: alertness, cognition, other mental function (eg. paranoia, hallucinations) arising over the last 2 weeks and still evident in last $24 \mathrm{hrs}$

Fig 1. Screening for delirium using the 4AT. Reproduced with permission from Maclullich et al. ${ }^{18}$

\section{4 or above: possible delirium +/- cognitive impairment 1-3: possible cognitive impairment 0: delirium or severe cognitive impairment unlikely (but delirium still possible if [4] information incomplete)}

\section{AT SCORE} suggests delirium but is not diagnostic: more detailed assessment of mental status may be required to reach a diagnosis. A score of 1-3 suggests cognitive impairment and more detailed cognitive testing and informant history-taking are required. A score of 0 does not definitively exclude delirium or cognitive impairment: more detailed testing may be required depending on the clinical context. Items 1-3 are rated solely on observation of the patient at the time of assessment. Item 4 requires information from one or more source(s), eg. your own knowledge of the patient, other staff who know the patient (eg. ward nurses), GP letter, case notes, carers. The tester should take account of communication difficulties (hearing impairment, dysphasia, lack of common language) when carrying out the test and interpreting the score.

Alertness: Altered level of alertness is very likely to be delirium in general hospital settings. If the patient shows significant altered alertness during the bedside assessment, score 4 for this item. AMT4 (Abbreviated Mental Test - 4): This score can be extracted from items in the AMT10 if the latter is done immediately before. Acute Change or Fluctuating Course: Fluctuation can occur without delirium in some cases of dementia, but marked fluctuation usually indicates delirium. To help elicit any hallucinations and/or paranoid thoughts ask the patient questions such as, "Are you concerned about anything going on here?"; "Do you feel frightened by anything or anyone?"; "Have you been seeing or hearing anything unusual?"

Over-reliance on investigation in place of a thorough collateral history and careful examination can cause diagnostic delay, and the transfer across the hospital for multiple tests can cause added harm. ${ }^{23}$ Constipation, urinary retention and medications are common causes of delirium that may not cause abnormality in blood tests. Investigations should be pragmatic with the routine use of simple tests (Box 4). A stepwise approach to further investigation is best directed by the clinical history and examination findings.

\section{Management}

Review daily

Only by prioritising cognitive state alongside vital signs in handovers will clinicians become sufficiently aware to pick up new change. Screening for and modifying known risk factors for delirium has been shown to prevent incident delirium in approximately one third of patients across a variety of care settings and healthcare systems. ${ }^{7,24}$ Methods recommended by 


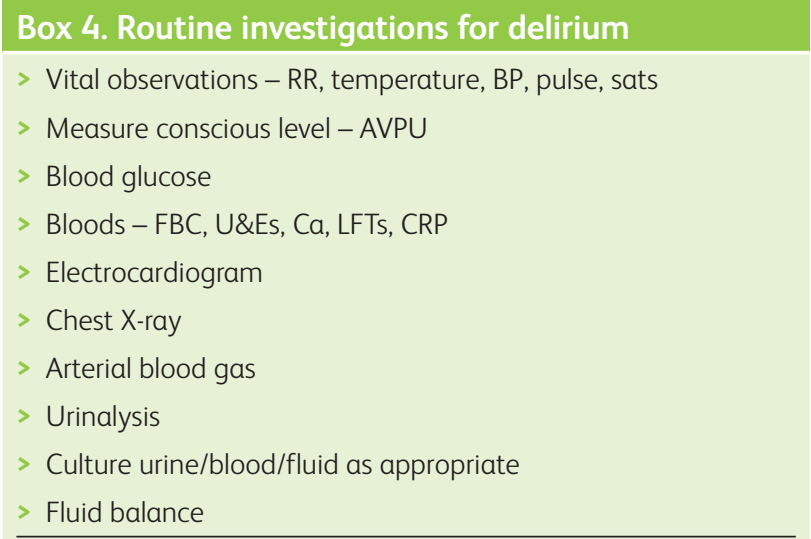

$\mathrm{AVPU}=$ conscious level score where $\mathrm{A}=$ alert, $\mathrm{V}=$ voice-responsive, $\mathrm{P}=$ pain-responsive and $\mathrm{U}=$ unresponsive; $\mathrm{BP}=$ blood pressure; $\mathrm{Ca}=$ calcium; $\mathrm{CRP}=\mathrm{C}$-reactive protein; $\mathrm{FBC}=$ full blood count; $\mathrm{LFTs}=$ liver biochemistry; $\mathrm{RR}=$ respiratory rate; sats $=$ oxygen saturations; $\mathrm{U \& Es}=$ urea and electrolytes
NICE are neither expensive nor time consuming, and broadly describe compassionate, patient-centred care. ${ }^{25}$ See Table 2 for practical examples. ${ }^{26-30}$

\section{Treat symptoms}

Patients with very agitated behaviour should be managed using the least restrictive options. Asking family for information regarding a patient's usual habits or familiar routines might help. Provision of a side room, one-to-one care and the encouragement of visiting by family are useful interventions. ${ }^{31}$ Good practice recommends regular engagement and communication with patient and family regarding changes to medication and care, and effort should be made to regularly assess capacity. Patients should be regularly asked about psychotic symptoms, distressing thoughts, delusional perceptions or hallucinations in a sensitive manner. When symptoms are particularly distressing or when patients present a risk to self or others and non-pharmacological means have been

\section{Table 2. Applications of NICE guideline CG103 on management of delirium ${ }^{25}$}

\section{Clinical factor}

Cognition and/or disorientation

\begin{tabular}{|c|c|}
\hline & Frequent orientation \\
\hline & Cognitively stimulating a \\
\hline & Access to family \\
\hline Dehydration and & Adequate hydration \\
\hline & Consideration of comorb \\
\hline & Regular bowel movemen \\
\hline Hypoxia & Optimisation of oxygeno \\
\hline Infection & Active search for and ear \\
\hline & Avoidance of unnecessar \\
\hline & Infection control \\
\hline Immobility & Early mobilisation \\
\hline & Access to walking aids \\
\hline Pain & Regular assessment for \\
\hline & Appropriate analgesia \\
\hline & Exclusion of hidden so \\
\hline Medication review & Medicine consolidation \\
\hline Nutrition & Well-fitting dentures \\
\hline & Adequate nutrition \\
\hline & Safe swallow \\
\hline $\begin{array}{l}\text { Sensory } \\
\text { impairment }\end{array}$ & Quiet at night \\
\hline
\end{tabular}

\section{Examples}

Appropriate lighting, clear signage, clock, calendar

Introduce oneself ('Hello My Name is...'), ${ }^{26}$ photos of family/ memorabilia at bedside, empower whole team approach

Therapy, volunteer befrienders, music consoles ${ }^{27}$

John's campaign to increase ward access to care-givers ${ }^{28}$

Volunteers to sit and eat with patients at mealtimes, supplementation of intravenous or subcutaneous fluids if required Regular assessment for fluid overload

Regularly asking about bowels, if necessary rectal exam to exclude impaction

Spacers for inhalers or nebuliser adjuncts; titrated oxygen therapy

Consider monitoring urine output via other means

Use antimicrobials with low risk of $C$ difficile, and eradicate MRSA in at-risk patients

Early discharge with enhanced home care teams where available ${ }^{29}$

Assess non-verbal signs of pain, eg using the Abbey Pain Scale ${ }^{30}$

Routinely examine for pressure sores, back pain and bladder retention

Review risk/benefit balance of primary and secondary prophylaxis

Consider dietary supplements, vitamin D deficiency

Avoid evening caffeinated drinks, schedule medication/ interventions around patient's natural sleep cycle, reduce noise to a minimum at night

Hearing aids that work and spectacles that are not lost

C difficile $=$ Clostridium difficile: MRSA = methicillin resistant Staphylococcus aureus; NICE = National Institute for Health and Care Excellence 
ineffective or are inappropriate, consider medical therapy. ${ }_{32}{ }^{25}$ There is evidence that benzodiazepines may cause harm. ${ }^{32}$ NICE guidelines ${ }^{25}$ and international consensus ${ }^{33}$ recommend anti-psychotic treatment at the lowest clinically appropriate dose, then titrated upwards, for no more than 1 week. Parkinson's disease and Lewy body dementia are important contraindications and specialist advice should be sought.

\section{Referral for specialist advice}

Movement of patients from one ward to the next may exacerbate or precipitate delirium, so should be avoided if possible. If the delirium persists despite measures detailed above, consider referral to a geriatrician or liaison psychiatrist specialising in care of older people. The expertise of liaison psychiatry is particularly indicated where there is risk of harm to self or others; persistent need of one-to-one supervision; or in cases of agitated behaviour or distressing psychotic symptoms not responding to first-line therapy.

\section{Follow-up}

Prognosis can be difficult to predict in delirium. While median duration of delirium is reported as 1 week, ${ }^{34}$ delirium may not fully resolve until the patient returns to their home environment, and for one third of patients symptoms persist 3 months later. ${ }^{35}$ An episode of delirium is often an isolated incident, and yet a proportion of patients will never fully recover their pre-delirium cognitive baseline and there is an association with future dementia risk. ${ }^{36}$ In the context of this uncertainty, routine follow up of patients with delirium is vital to support patient and caregivers and to optimise recovery. Clear documentation and coding of delirium diagnosis are therefore essential in communications between secondary and primary care at discharge.

\section{Conclusion}

Delirium is common but remains neglected and underdiagnosed despite its significant impact upon the health of older people. Early identification of those most at risk can enable targeted methods to effectively prevent the evolution of delirium. There is a need for further research to better understand causal mechanisms as well as develop a stronger evidence base for treatment strategies. In the meantime, clinical practice must change in order to apply effective delirium prevention techniques.

\section{Conflicts of interest}

Oliver Todd is funded for by an academic clinical fellowship at the National Institute of Health Research.

\section{References}

1 Siddiqi N, House AO, Holmes JD. Occurrence and outcome of delirium in medical in-patients: a systematic literature review. Age Ageing 2006;35:350-64.

2 Ryan DJ, O'Regan NA, Caoimh RO et al. Delirium in an adult acute hospital population: predictors, prevalence and detection. BMJ Open 2013;3:e001772.

3 Collins N, Blanchard MR, Tookman A, Sampson EL. Detection of delirium in the acute hospital. Age Ageing 2009;39:131-5.

4 Kakuma R, du Fort GG, Arsenault L et al. Delirium in older emergency department patients discharged home: effect on survival. J Am Geriatr Soc 2003;51:443-50.
5 Witlox J, Eurelings LS, de Jonghe JF et al. Delirium in elderly patients and the risk of postdischarge mortality, institutionalization, and dementia: a meta-analysis. JAMA 2010;304:443-51.

6 Kerr CW, Donnelly JP, Wright ST et al. Progression of delirium in advanced illness: a multivariate model of caregiver and clinician perspectives. J Palliat Med 2013;16:768-73.

7 Hshieh TT, Yue J, Oh E et al. Effectiveness of multicomponent nonpharmacological delirium interventions: a meta-analysis. JAMA Intern Med 2015;175:512-20.

8 Siddiqi N CA, Young J. Delirium in care homes. Rev Clin Gerontol 2009;19:309-16.

9 Young J, Murthy L, Westby M, Akunne A, O’Mahony R. Diagnosis, prevention, and management of delirium: summary of NICE guidance. BMJ 2010;341:c3704.

10 Davis DH, Skelly DT, Murray C et al. Worsening cognitive impairment and neurodegenerative pathology progressively increase risk for delirium. Am J Geriatr Psychiatry 2014;23:403-15.

11 Jackson TA, MacLullich AM, Gladman JR, Lord JM, Sheehan B. Diagnostic test accuracy of informant-based tools to diagnose dementia in older hospital patients with delirium: a prospective cohort study. Age Ageing 2016;45:505-11.

12 Patient. Informant Questionnaire on Cognitive Decline in the Elderly (IQCODE). http://patient.info/doctor/informant-questionnaireon-cognitive-decline-in-the-elderly-iqcode [Accessed 21 September 2016].

13 European Delirium Association, American Delirium Society. The DSM-5 criteria, level of arousal and delirium diagnosis: inclusiveness is safer. BMC Med 2014;12:141.

14 American Psychiatric Association (ed). Diagnostic and statistical manual of mental disorders, 5th edn. Arlington: American Psychiatric Association, 2013.

15 Sands MB, Dantoc BP, Hartshorn A, Ryan CJ, Lujic S. Single Question in Delirium (SQiD): testing its efficacy against psychiatrist interview, the Confusion Assessment Method and the Memorial Delirium Assessment Scale. Palliat Med 2010;24:561-5.

16 O'Regan NA, Ryan DJ, Boland E et al. Attention! A good bedside test for delirium? J Neurol Neurosurg Psychiatry 2014;85:1122-31.

17 Bellelli G, Morandi A, Davis DH et al. Validation of the 4AT, a new instrument for rapid delirium screening: a study in 234 hospitalised older people. Age Ageing 2014;43:496-502.

18 Maclullich A, Ryan T, Cash H. 4AT: rapid assessment test for delirium, 2011. www.the4at.com/ [Accessed 21 September 2016].

19 Meagher DJ, Leonard M, Donnelly S et al. A longitudinal study of motor subtypes in delirium: relationship with other phenomenology, etiology, medication exposure and prognosis. J Psychosom Res 2011;71:395-403.

20 Holt R, Teale EA, Mulley GP, Young J. A prospective observational study to investigate the association between abnormal hand movements and delirium in hospitalised older people. Age Ageing 2014;44:42-5.

21 American Geriatrics Society. 2012 Beers Criteria Update Expert Panel. American Geriatrics Society updated Beers Criteria for potentially inappropriate medication use in older adults. $J \mathrm{Am}$ Geriatr Soc 2012;60:616-31.

22 Clegg A, Young JB. Which medications to avoid in people at risk of delirium: a systematic review. Age Ageing 2010;40;23-9.

23 Goldberg A, Straus SE, Hamid JS, Wong CL. Room transfers and the risk of delirium incidence amongst hospitalized elderly medical patients: a case-control study. BMC Geriatr 2015;15:69.

24 Siddiqi N, Harrison JK, Clegg A et al. Interventions for preventing delirium in hospitalised non-ICU patients. Cochrane Database Syst Rev 2016;(3):CD005563.

25 National Institute for Health and Care Excellence. Delirium: prevention, diagnosis and management. NICE clinical guideline No 103. Manchester: NICE, 2010.

26 Granger K. \#hello my name is.., 2015. http://hellomynameis.org.uk/ [Accessed 21 September 2016]. 
27 Alive inside: a story of music and memory, 2014 [Documentary]. www.aliveinside.us/\#land [Accessed 21 September 2016].

28 Gerrard N. John's campaign, 2015. http://johnscampaign.org.uk/\#/ [Accessed 21 September 2016].

29 Angel T, Holland G, Mandell M et al. The Delirium Recovery Programme (DRP): pilot study of cognitive enablement at home following hospital admission. West Hertfordshire Hospitals Trust and Hertfordshire Partnership University NHS Foundation Trust, 2014. www.rcpsych. ac.uk/pdf/RAID\%20Delirium\%20Recovery\%20programme2.pdf [Accessed 21 September 2016].

30 Abbey J, Piller N, De Bellis A et al. The Abbey pain scale: a 1-minute numerical indicator for people with end-stage dementia. Int J Palliat Nurs 2004;10:6-13.

31 Royal College of Physicians. The prevention, diagnosis and management of delirium in older people. London: RCP, 2006.

32 Marcantonio ER, Juarez G, Goldman L et al. The relationship of postoperative delirium with psychoactive medications. JAMA 1994;272:1518-22.
33 Morandi A, Davis D, Taylor JK et al. Consensus and variations in opinions on delirium care: a survey of European delirium specialists. Int Psychogeriatr 2013;25:2067-75.

34 McCusker J, Cole M, Dendukuri N, Han L, Belzile E. The course of delirium in older medical inpatients: a prospective study. J Gen Intern Med 2003;18:696-704.

35 Cole MG. Persistent delirium in older hospital patients. Curr Opin Psychiatry 2010;23:250-4.

36 Davis DH, Muniz Terrera G, Keage $\mathrm{H}$ et al. Delirium is a strong risk factor for dementia in the oldest-old: a population-based cohort study. Brain 2012;135:2809-16.

\section{Address for correspondence: Dr O M Todd, Academic Unit of}

Elderly Care and Rehabilitation, University of Leeds, Bradford Institute for Health Research, Duckworth Lane, Bradford BD9 6RJ, UK.

Email: olitodd@gmail.com 